Proyecciones Journal of Mathematics

Vol. 31, $\mathrm{N}^{o}$ 2, pp. 125-147, June 2012.

Universidad Católica del Norte

Antofagasta - Chile

\title{
Some separation axioms in $L$-topological spaces
}

\author{
CUI-MEI JIANG \\ QINGDAO TECHNOLOGICAL UNIVERSITY, CHINA \\ and \\ $J I N-M I N G$ FANG \\ OCEAN UNIVERSITY OF CHINA, CHINA \\ Received : December 2011. Accepted : January 2012
}

\begin{abstract}
In this paper, under the idea of $L-T_{0}$ or $s u b-T_{0}$, we propose a set of new separation axioms in L-topological spaces, namely sub-separation axioms. And some of their properties are studied. In addition, the relation between the sub-separation axioms defined in the paper and other separation axioms is discussed. The results show that the subseparation axioms in this paper are weaker than other separation axioms that had appeared in literature.
\end{abstract}

Keywords : L-topology; sub-separation axioms; sub- $T_{1} ; s u b-T_{2} ; s u b$ $T_{2 \frac{1}{2}} ; s u b-T_{3} ; s u b-T_{4}$. 


\section{Introduction and preliminaries}

Since Chang [1] introduced fuzzy theory into topology, Wong, Lowen, Hutton etc., discussed respectively various aspects of fuzzy topology (Wong [17], Lowen [12], Hutton [6]).

Separation is an essential part of fuzzy topology, on which a lot of work have been done [2-19]. In 1983, Liu [9] introduced the sub- $T_{0}$ axiom, for underlying lattice $L$ being a completely distributive DeMorgan algebra, in terms of closed sets and proved that the fuzzy real line and the fuzzy unit interval satisfy this axiom. Wuyts and Lowen [18] and Rodabaugh [13] independently gave a more general $L-T_{0}$ axioms, the latter for $L$ being a complete lattice, using only open sets and equivalent to the sub- $T_{0}$ when $L$ is a completely distributive DeMorgan algebra. The aim of this paper is to study some separation axioms on the basis of the thought of the sub- $T_{0}$ and the layer of $L$-topology.

Now we recall some the concepts required in the sequel.

Throughout this paper, $\left(L, \bigvee, \wedge,,^{\prime}\right)$ is a completely distributive DeMorgan algebra, i.e., a complete and completely distributive lattice with an order-reversing involution ()$^{\prime}$, and with the smallest element $\perp$ and the largest element $\top(\perp \neq \top)$. Obviously, for every nonempty set $X, L^{X}$, the family of all $L$-sets, i.e., all mappings from $X$ to $L$, is also a complete and completely distributive lattice under the pointwise order. we denote the smallest element and the largest element of $L^{X}$ by $\perp_{X}$ and $\top_{X}$, respectively. For any $A \in L^{X}$, the set $\{x: A(x) \neq \perp\}$ is called the support of $A$ and denoted by $\operatorname{supp} A$, i.e., $\operatorname{supp} A=\{x: A(x) \neq \perp\}$

An $L$-topological space, briefly $L$-ts, is a pair $\left(L^{X}, \delta\right)$, where $\delta$, called an $L$-topology on $L^{X}$, a subfamily of $L^{X}$ closed under the operation of finite intersections and arbitrary unions, and $\delta^{\prime}=\left\{A^{\prime}: A \in \delta\right\}$; the member of $\delta$ (resp. $\left.\delta^{\prime}\right)$ is called open (resp., closed) $L$-sets, and for each $B \in L^{X}$, the $L$-set $B^{\circ}=\bigvee\{U \in \delta: U \leq B\} \quad\left(\right.$ resp. $B^{-}=\bigwedge\left\{C \in \delta^{\prime}: B \leq C\right\}$ ) is called the interior (resp., closure) of $B$. An element $\lambda \in L$ is called a molecule if $\lambda \neq \perp$ and $\lambda \leq a \vee b$ implies $\lambda \leq a$ or $\lambda \leq b$. The set of all molecules of $L$ (resp., $L^{X}$ ) will be denoted by $M(L)$ (resp., $M\left(L^{X}\right)$ ); obviously, $M\left(L^{X}\right)=\left\{x_{\lambda}: x \in X, \lambda \in M(L)\right\}$. For any $x_{\lambda} \in M\left(L^{X}\right)$, a closed $L$-set $P \in \delta^{\prime}$ is called a closed remote neighborhood of $x_{\lambda}$ if $x_{\lambda} \not \leq P$. The set of all closed remote neighborhood of $x_{\lambda}$ is denoted by $\eta^{-}\left(x_{\lambda}\right)$. For any $A \in L^{X}$, a closed $L$-set $P \in \delta^{\prime}$ is called a closed remote neighborhood of $A$ if for any $x \in \operatorname{supp} A$ such that $A(x) \not \subset P(x)$. The set of all closed 
remote neighborhood of $A$ is denoted by $\eta^{-}(A)$. For any $A \in L^{X}, A$ is a called pseudo-crisp closed set if $\exists a \in L-\{\perp\}$ such that $A(x)>\perp$ if and only if $\forall x \in X, A(x) \geq a$.

Let $f: X \longrightarrow Y$ be an ordinary mapping. Based on $f: X \longrightarrow Y$ define an mapping $f^{\rightarrow}: L^{X} \longrightarrow L^{Y}$ which is called a function of Zadeh's type and its right adjoint mapping $f^{\leftarrow}: L^{Y} \longrightarrow L^{X}$ by

$$
\begin{gathered}
\forall A \in L^{X}, \forall y \in Y, \quad f^{\rightarrow}(A)(y)=\bigvee\{A(x): x \in X, f(x)=y\}, \text { and } \\
\forall B \in L^{Y}, \forall x \in X, \quad f^{\leftarrow}(B)(x)=B(f(x)), \text { respectively. }
\end{gathered}
$$

For other undefined notions and symbols in this paper, please refer to Wang [16].

Definition 1.1 (Liu [9]). An $L$-ts $\left(L^{X}, \delta\right)$ is called a sub- $T_{0}$ space if for any $x, y \in X$ with $x \neq y$, there exists $\lambda \in M(L)$, either there is $P \in \eta^{-}\left(x_{\lambda}\right)$ such that $y_{\lambda} \leq P$ or there is $Q \in \eta^{-}\left(y_{\lambda}\right)$ such that $x_{\lambda} \leq Q$.

Definition 1.2 (Chen and Meng [2]). An $L$-ts $\left(L^{X}, \delta\right)$ is called a $T_{2 \frac{1}{2}}$ or $L$-Urysohn space if for any $x_{\lambda}, y_{\mu} \in M\left(L^{X}\right)$ with $x \neq y$, there exist $P \in \eta^{-}\left(x_{\lambda}\right)$ and $Q \in \eta^{-}\left(y_{\mu}\right)$ such that $P^{\circ} \vee Q^{\circ}=\top_{X}$.

Definition 1.3 (Wang [16]). Let $\left(L^{X}, \delta\right)$ be an $L$-ts. Then,

(1) $\left(L^{X}, \delta\right)$ is said to be $T_{1}$ if for any $x_{\lambda}, y_{\mu} \in M\left(L^{X}\right)$ with $x_{\lambda} \not \leq y_{\mu}$, there exists $P \in \eta^{-}\left(x_{\lambda}\right)$ such that $y_{\mu} \leq P$.

(2) $\left(L^{X}, \delta\right)$ is said to be $T_{2}$ (or Hausdorff) if for any $x_{\lambda}, y_{\mu} \in M\left(L^{X}\right)$ with $x_{\lambda} \not \leq y_{\mu}$, there exist $P \in \eta^{-}\left(x_{\lambda}\right)$ and $Q \in \eta^{-}\left(y_{\mu}\right)$ such that $P \vee Q=\top_{X}$.

(3) $\left(L^{X}, \delta\right)$ is said to be regular if for each $x_{\lambda} \in M\left(L^{X}\right)$ and each nonempty pseudo-crisp closed set $A$ with $x \notin \operatorname{supp} A$, there exist $P \in \eta^{-}\left(x_{\lambda}\right)$ and $Q \in \eta^{-}(A)$ such that $P \vee Q=\top_{X} .\left(L^{X}, \delta\right)$ is said to be $T_{3}$ if it is regular and $T_{1}$.

(4) $\left(L^{X}, \delta\right)$ is said to be normal if for each pair of nonempty pseudo-crisp closed set $A$ and $B$ with supp $A \cap \operatorname{supp} B=\varnothing$, there exist $P \in \eta^{-}(A)$ and $Q \in \eta^{-}(B)$ such that $P \vee Q=\top_{X} .\left(L^{X}, \delta\right)$ is said to be $T_{4}$ if it is normal and $T_{1}$.

Theorem 1.4 (Wang [16], You [19]). $T_{i}\left(i=1,2,2 \frac{1}{2}, 3,4\right)$ is $L$-good extension in Lowen's sense.

Definition 1.5 ( $\mathrm{Gu}$ and Zhao [4]). An $L$-ts $\left(L^{X}, \delta\right)$ is said to be layer $T_{0}$ if for any $\alpha \in M(L),\left(X,\left(\tau_{\alpha}\left(\delta^{\prime}\right)\right)^{\prime}\right)$ is $T_{0}$, where $\tau_{\alpha}(\delta)=\left\{\tau_{\alpha}(A): A \in\right.$ 
$\left.\delta^{\prime}\right\}, \tau_{\alpha}(A)=\{x \in X: A(x) \geq \alpha\}$. In the same way, layer $T_{i}(i=1,2,3,4)$ and layer regular (completely regular, normal) are defined.

Definition 1.6 (Kubiak [7]). An $L$-ts $\left(L^{X}, \delta\right)$ is said to be

(i) Kubiak- $T_{1}$ (or $L-T_{1}$ ) if for all $x, y \in X$ with $x \neq y$, there exist $U, V \in \delta$ such that $U(x) \not \leq U(y)$ and $V(y) \not Z V(x)$.

(ii) Kubiak- $T_{2}$ if for all $x, y \in X$ with $x \neq y$, there exist $U, V \in \delta$ such that $U(x) \not \leq U(y), V(y) \not \leq V(x)$ and $U \leq V^{\prime}$.

Lemma 1.7 (Liu and Luo [10]). Let $\left(L^{X}, \delta\right)$ be an $L$-ts, where $\delta$ is generated by a classical topology, then for any $A \in L^{X}$ such that $A^{\circ}=$ $\bigvee\left\{\alpha \chi_{\left[\tau_{\alpha}(A)\right]^{\circ}}: \alpha \in M(L)\right\}$.

Definition 1.8 (Shi [14]). An $L$-ts $\left(L^{X}, \delta\right)$ is called $L-T_{2}$ if for all $x, y \in X$ with $x \neq y$, there exist $P \in \delta^{\prime}$ and $Q \in \delta$ such that $Q \leq P$ and $Q(x) \not \subset P(y)$.

\section{Definitions and characterizations}

In this section, we introduce the concept of sub- $T_{1}$, sub- $T_{2}$, sub- $T_{2 \frac{1}{2}}$, sub- $T_{3}$ and sub- $T_{4}$ separation axioms in $L$ - topological spaces and establish the characteristic theorems of these sub-separation axioms. First, some definitions are given as follows:

Definition 2.1. Suppose that $\left(L^{X}, \delta\right)$ is an $L$-ts. Then,

(1) $\left(L^{X}, \delta\right)$ is said to be sub- $T_{1}$ if for any $x, y \in X$ with $x \neq y$, there exists $\lambda \in M(L)$, both there is $P \in \eta^{-}\left(x_{\lambda}\right)$ such that $y_{\lambda} \leq P$ and there is $Q \in \eta^{-}\left(y_{\lambda}\right)$ such that $x_{\lambda} \leq Q$.

(2) $\left(L^{X}, \delta\right)$ is said to be sub- $T_{2}$ if for any $x, y \in X$ with $x \neq y$, there exists $\lambda \in M(L)$, and there are $P \in \eta^{-}\left(x_{\lambda}\right)$ and $Q \in \eta^{-}\left(y_{\lambda}\right)$ such that $P \vee Q=\top_{X}$.

(3) $\left(L^{X}, \delta\right)$ is said to be sub- $T_{2 \frac{1}{2}}$ if for any $x, y \in X$ with $x \neq y$, there exists $\lambda \in M(L)$, and there are $P \in \eta^{-}\left(x_{\lambda}\right)$ and $Q \in \eta^{-}\left(y_{\lambda}\right)$ such that $P^{\circ} \vee Q^{\circ}=\top_{X}$.

(4) $\left(L^{X}, \delta\right)$ is said to be sub-regular if for each $x \in X$ and each nonempty pseudo-crisp closed set $A$ with $x \notin \operatorname{supp} A$, there exists $\lambda \in M(L)$, and there are $P \in \eta^{-}\left(x_{\lambda}\right)$ and $Q \in \eta^{-}(\lambda A)$ such that $P \vee Q=\top_{X} .\left(L^{X}, \delta\right)$ is said to be sub- $T_{3}$ if it is sub-regular and sub- $T_{1}$.

(5) $\left(L^{X}, \delta\right)$ is said to be sub-normal if for each pair of nonempty pseudo- 
crisp closed set $A$ and $B$ with supp $A \cap \operatorname{supp} B=\varnothing$, there exists $\lambda \in M(L)$, and there are $P \in \eta^{-}(\lambda A)$ and $Q \in \eta^{-}(\lambda B)$ such that $P \vee Q=\top_{X}$, where $\lambda A(x)=\lambda \wedge A(x)$ for any $x \in X . \quad\left(L^{X}, \delta\right)$ is said to be sub- $T_{4}$ if it is sub-normal and sub- $T_{1}$.

By Definition 2.1, we have:

Corollary 2.2. The following implications hold: $T_{i} \Longrightarrow$ sub- $T_{i}$, where $i=$ $1,2,2 \frac{1}{2}, 3,4$.

Now we introduce the convergence of molecular nets. Let $\left(L^{X}, \delta\right)$ be an $L$-ts, $S=\{S(n): n \in D\}$ a molecular net and $e \in M\left(L^{X}\right)$, $e$ is said to be a limit point of $S$, (or $S$ converges to $e$; in symbols, $S \rightarrow e$ ), if for $\forall P \in \eta^{-}(e), S(n) \not \leq P$ is eventually true, that is there exists $m \in D$ such that $S(n) \not \leq P$ for all $n \in D$ with $n \geq m$. The following results show that the convergence of molecular nets is unique under a certain condition for the sub- $T_{2}$ space.

Theorem 2.3. Let $\left(L^{X}, \delta\right)$ be a sub- $T_{2}$ space, then for each molecular net $S$ such that $\left|K_{S}\right| \leq 1$, where $K_{S}=\{x \in X: \lim S(x)=\top\}$.

Proof. Let $\left(L^{X}, \delta\right)$ be a sub- $T_{2}$ space and $S=\{S(n): n \in D\}$ be a molecular net. Assume that $\left|K_{S}\right| \geq 2$, for any $x, y \in K_{S}$ with $x \neq y$, since $\left(L^{X}, \delta\right)$ is sub- $T_{2}$, there exists $\lambda \in M(L)$, and there are $P \in \eta^{-}\left(x_{\lambda}\right)$ and $Q \in \eta^{-}\left(y_{\lambda}\right)$ such that $P \vee Q=\top_{X}$. Then we have $S \rightarrow x_{\lambda}$ and $S \rightarrow y_{\lambda}$ from $x_{\lambda} \leq \lim S$ and $y_{\lambda} \leq \lim S$ by Theorem 2.3.4 (Wang [16]). So there exists an $n_{1} \in D$ such that $S(n) \not \geq P$ for all $n \in D$ with $n \geq n_{1}$ and there exists an $n_{2} \in D$ such that $S(n) \not \leq Q$ for all $n \in D$ with $n \geq n_{2}$. Taking $n_{3} \in D$ such that $n_{3} \geq n_{1}$ and $n_{3} \geq n_{2}$, hence we have $S(n) \not \leq P \vee Q$ when $n \geq n_{3}$.This implies that we must have $P \vee Q \neq \top_{X}$. This is a contradiction.

If $T$ is a molecule, the inverse of Theorem 2.3 is also true.

Theorem 2.4. $\left(L^{X}, \delta\right)$ is an $L$-ts, if for each molecular net $S$ with $\left|K_{S}\right| \leq 1$, where $K_{S}=\{x \in X: \lim S(x)=\top\}$, then $\left(L^{X}, \delta\right)$ is a sub- $T_{2}$ space.

Proof. Suppose that $\left(L^{X}, \delta\right)$ is not a sub- $T_{2}$ space, then there exist $x, y \in X$ satisfying $x \neq y, \forall \lambda \in M(L), \forall P \in \eta^{-}\left(x_{\lambda}\right)$ and $\forall Q \in \eta^{-}\left(y_{\lambda}\right)$ such that $P \vee Q \neq \top_{X}$. Let $D(\lambda)=\eta^{-}\left(x_{\lambda}\right) \times \eta^{-}\left(y_{\lambda}\right)$ and $D(\lambda)$ be a directed set by 
product order. For each $m=(P, Q) \in D(\lambda)$, we can take a molecule $S^{\lambda}(m)$ such that $S^{\lambda}(m) \not \leq P \vee Q$. Let $S^{\lambda}=\left\{S^{\lambda}(m): m \in D(\lambda)\right\}$, hence it is easy to prove $S^{\lambda} \rightarrow x_{\lambda}$ and $S^{\lambda} \rightarrow y_{\lambda}$. Therefore, $\lim S^{\lambda} \geq x_{\lambda} \vee y_{\lambda}$. Since $\top$ is a molecule, the standard minimal set $\beta^{*}(\top)$ is a directed set(Wang [16]). We denote $\beta^{*}(\top)$ by $E$, i.e., $E=\beta^{*}(\top)$. Noticing that $\left\{x_{\lambda}\right\}_{\lambda \in E},\left\{y_{\lambda}\right\}_{\lambda \in E}$ are molecular nets and $\left\{x_{\lambda}\right\}_{\lambda \in E} \rightarrow x_{\top},\left\{y_{\lambda}\right\}_{\lambda \in E} \rightarrow y_{\top}$, we can make a molecular net $\bar{S}: E \times \prod_{\lambda \in E} D(\lambda) \longrightarrow M\left(L^{X}\right)$ such that

$$
\bar{S}(\lambda, f)=S^{\lambda}(f(\lambda)), \quad \forall(\lambda, f) \in E \times \prod_{\lambda \in E} D(\lambda) .
$$

Then, $\bar{S} \rightarrow x_{\top}, \quad \bar{S} \rightarrow y_{\top}$. In fact, for every $P \in \eta^{-}\left(x_{\top}\right)$, since $\left\{x_{\lambda}\right\}_{\lambda \in E} \rightarrow x_{\top}$, there exists a $\lambda_{0} \in E$ such that $x_{\lambda} \not \subset P$ for all $\lambda \in E$ with $\lambda \geq \lambda_{0}$. It follows from $S^{\lambda} \rightarrow x_{\lambda}$ for $\lambda \in E$ that there exists $m_{\lambda} \in D(\lambda)$ s.t. $S^{\lambda}(m) \not \leq P$ for all $m \in D(\lambda)$ with $m \geq m_{\lambda}$. We define $f_{0} \in \prod_{\lambda \in E} D(\lambda)$ as follows:

$$
f_{0}(\lambda)= \begin{cases}m_{\lambda}, & \lambda \leq \lambda_{0} . \\ \text { any fixed element in } D(\lambda), & \lambda \not \subset \lambda_{0} .\end{cases}
$$

Then we can prove for every pair $(\lambda, f) \in E \times \prod_{\lambda \in E} D(\lambda)$ with $(\lambda, f) \geq$ $\left(\lambda_{0}, f_{0}\right)$ such that $\bar{S}(\lambda, f) \not \subset P$, i.e., $\bar{S}$ is not in any closed remote neighborhood $P$ of $x_{\top}$ eventually. So we have $\bar{S} \rightarrow x_{\top}$. Similarly, we can prove $\bar{S} \rightarrow y_{\top}$. Therefore, $\left|K_{\bar{S}}\right| \geq 2$. This contradicts to $\left|K_{\bar{S}}\right| \leq 1$. Thus, we conclude that $\left(L^{X}, \delta\right)$ is a sub- $T_{2}$ space.

With Theorem 2.3 and Theorem 2.4, we have:

Corollary 2.5. Let $\top$ be a molecule, then $\left(L^{X}, \delta\right)$ is a sub- $T_{2}$ space iff for each molecular net $S$ such that $\left|K_{S}\right| \leq 1$, where $K_{S}=\{x \in X: \lim S(x)=$ T\}.

For the sub- $T_{2}$ space, we have the following theorem:

Theorem 2.6. Let $\left(L^{X}, \delta\right)$ be a sub- $T_{2}$ space and $T$ be a molecule, then super F-compactness, N-compactness, strongly F-compactness and F-compactness are equivalent. 
Proof. The proof is similar to that of Wang's Theorem 6.4.29 in [16].

Theorem 2.7. Suppose that $\left(L^{X}, \delta\right)$ is a weakly induced $L$-ts. If it is a sub- $T_{2}$ space, then $(X,[\delta])$ is a $T_{2}$ space.

Proof. Let $\left(L^{X}, \delta\right)$ be a sub- $T_{2}$ space and $x, y \in X$ with $x \neq y$. Then there exists $\lambda \in M(L)$, and there are $P \in \eta^{-}\left(x_{\lambda}\right)$ and $Q \in \eta^{-}\left(y_{\lambda}\right)$ such that $P \vee Q=\top_{X}$. We put

$$
\begin{gathered}
U=\left\{t \in X: P^{\prime}(t) \not \lambda^{\prime}\right\}=\{t \in X: P(t) \nsupseteq \lambda\}, \text { and } \\
V=\left\{t \in X: Q^{\prime}(t) \not \leq \lambda^{\prime}\right\}=\{t \in X: Q(t) \nsupseteq \lambda\} .
\end{gathered}
$$

Then it is easy to know that $\chi_{U}, \chi_{V} \in \delta$ i.e., $U, V \in[\delta]$. Obviously $x \in U, y \in V$. Thus it remains only to show that $U \cap V=\varnothing$. In fact, if there were a $z \in U \cap V$, then we have $\lambda \not \leq P(z)$ and $\lambda \not \leq Q(z)$. Hence, $\lambda \not \leq(P \vee Q)(z)$, which contradicts to $P \vee Q=\top_{X}$. Therefore, $(X,[\delta])$ is a $T_{2}$ space.

\section{Properties}

In this section, we will investigate some nice properties of sub-separation axioms. At first, we show that sub-separation axioms are good extensions in the sense of Lowen.

Theorem 3.1. Let $(X, \mathcal{T})$ be a crisp topological space. Then $\left(L^{X}, \omega_{L}(\mathcal{T})\right)$ is a sub- $T_{i}$ space iff $(X, \mathcal{T})$ is a $T_{i}$ space, where $i=1,2,2 \frac{1}{2}, 3,4$.

Proof. Sufficiency. Let $(X, \mathcal{T})$ be a $T_{i}$ space $\left(i=1,2,2 \frac{1}{2}, 3,4\right)$. Then $\left(L^{X}, \omega_{L}(\mathcal{T})\right)$ is a $T_{i}$ space by Theorem 1.4. Thus from Corollary 2.2, we know that $\left(L^{X}, \omega_{L}(\mathcal{T})\right)$ is a sub- $T_{i}$ space.

Necessity. Case $i=1$ : Let $\left(L^{X}, \omega_{L}(\mathcal{T})\right)$ be a sub- $T_{1}$ space. For any $x \in X$ and taking $y \in X$ with $x \neq y$, since $\left(L^{X}, \omega_{L}(\mathcal{T})\right)$ is a sub- $T_{1}$ space, there exists $\lambda \in M(L)$, both there is $P \in \eta^{-}\left(y_{\lambda}\right)$ such that $x_{\lambda} \leq P$ and there is $Q \in \eta^{-}\left(x_{\lambda}\right)$ such that $y_{\lambda} \leq Q$. We put

$$
U=\left\{z \in X: P^{\prime}(z) \not \subset \lambda^{\prime}\right\} .
$$

It is clear that $U \in \mathcal{T}, x \notin U$ and $y \in U$. Hence $y \notin\{x\}^{-}$, where $\{x\}^{-}$is the closure of $\{x\}$. Therefore, $(X, \mathcal{T})$ is a $T_{1}$ space. 
Case $i=2$ : For any two distinct points $x, y \in X$ with $x \neq y$, since $\left(L^{X}, \omega_{L}(\mathcal{T})\right)$ is a sub- $T_{2}$ space, there exists $\lambda \in M(L)$, and there are $P \in$ $\eta^{-}\left(x_{\lambda}\right)$ and $Q \in \eta^{-}\left(y_{\lambda}\right)$ such that $P \vee Q=\top_{X}$. We put

$$
U=\left\{z \in X: P^{\prime}(z) \not \subset \lambda^{\prime}\right\}, \quad V=\left\{z \in X: Q^{\prime}(z) \not \subset \lambda^{\prime}\right\} .
$$

Noticing that $P^{\prime}, Q^{\prime} \in \omega_{L}(\mathcal{T})$ and $x_{\lambda} \not \leq P, y_{\lambda} \not \leq Q$, hence $U, V \in \mathcal{T}$ and $x \in U, y \in V$. Thus it remains only to show that $U \cap V=\varnothing$. In fact, if there were a $z \in U \cap V \neq \varnothing$, then we have $\lambda \not \leq P(z)$ and $\lambda \not \leq Q(z)$. Hence $\lambda \not \leq(P \vee Q)(z)=\top$, which contradicts to $\lambda \leq \top$. Therefore, $(X, \mathcal{T})$ is a $T_{2}$ space.

Case $i=2 \frac{1}{2}$ : For any $x, y \in X$ with $x \neq y$, since $\left(L^{X}, \omega_{L}(\mathcal{T})\right)$ is a sub- $T_{2} \frac{1}{2}$ space, there exists $\lambda \in M(L)$, and there are $P \in \eta^{-}\left(x_{\lambda}\right)$ and $Q \in \eta^{-}\left(y_{\lambda}\right)$ such that $P^{\circ} \vee Q^{\circ}=\top_{X}$. Clearly, $P \vee Q=\top_{X}$. From $\lambda \not \leq P(x)$ and $\lambda \not \leq Q(y)$, we know that there exist $\lambda_{P} \in \beta^{*}(\lambda)$ and $\lambda_{Q} \in \beta^{*}(\lambda)$ such that $\lambda_{P} \not \leq P(x)$ and $\lambda_{Q} \not \leq Q(y)$. Since $\lambda$ is a molecule, the standard minimal set $\beta^{*}(\lambda)$ is a directed set (Wang [16]). Taking $\gamma \in \beta^{*}(\lambda)$ such that $\gamma \geq \lambda_{P} \vee \lambda_{Q}$. We put

$$
E=\tau_{\gamma}(P)=\{z: P(z) \geq \gamma\}, F=\tau_{\gamma}(Q)=\{z: Q(z) \geq \gamma\} .
$$

It is clear that $E, F \in \mathcal{T}^{\prime}, x \notin E, y \notin F$ and $E \cup F=X$. In order to prove that $(X, \mathcal{T})$ is a $T_{2} \frac{1}{2}$ space, we need only to verify $E^{\circ} \cup F^{\circ}=X$ by the definition of $T_{2} \frac{1}{2}$. For this purpose, we firstly prove that $\tau_{\lambda}\left(P^{\circ}\right) \subseteq\left[\tau_{\gamma}(P)\right]^{\circ}$ and $\tau_{\lambda}\left(Q^{\circ}\right) \subseteq\left[\tau_{\gamma}(Q)\right]^{\circ}$. In fact, taking $z \in \tau_{\lambda}\left(P^{\circ}\right)$, from Lemma 1.7, we have

$$
\bigvee_{\alpha \in M(L)} \alpha \chi_{\left[\tau_{\alpha}(P)\right]^{\circ}}(z)=P^{\circ}(z) \geq \lambda
$$

Therefore, there exists $\alpha \in M(L)$ such that $z \in\left[\tau_{\alpha}(P)\right]^{\circ}$ and $\alpha \geq \gamma$. i.e., $z \in\left[\tau_{\alpha}(P)\right]^{\circ} \subseteq\left[\tau_{\gamma}(P)\right]^{\circ}$. Hence, we obtain that $\tau_{\lambda}\left(P^{\circ}\right) \subseteq\left[\tau_{\gamma}(P)\right]^{\circ}$ from the arbitrariness of $z$. Similarly, we can get $\tau_{\lambda}\left(Q^{\circ}\right) \subseteq\left[\tau_{\gamma}(Q)\right]^{\circ}$, as desired. Naturally, we have

$$
E^{\circ} \cup F^{\circ}=\left[\tau_{\gamma}(P)\right]^{\circ} \cup\left[\tau_{\gamma}(Q)\right]^{\circ} \supseteq \tau_{\lambda}\left(P^{\circ}\right) \cup \tau_{\lambda}\left(Q^{\circ}\right)=\tau_{\lambda}\left(P^{\circ} \vee Q^{\circ}\right)=X,
$$

i.e., $(X, \mathcal{T})$ is a $T_{2 \frac{1}{2}}$ space.

Case $i=3$ : Since sub- $T_{1}$ separation axiom is an $L$-good extension, we prove this theorem only for the sub-regular case.

For any $x \in X$, suppose that $E \in \mathcal{T}^{\prime}$ with $x \notin E$. Clearly, $\chi_{E}$ is a nonempty pseudo-crisp closed set in $\left(L^{X}, \omega_{L}(\mathcal{T})\right)$ and $x \notin \operatorname{supp}\left(\chi_{E}\right)$. Since 
$\left(L^{X}, \omega_{L}(\mathcal{T})\right.$ is a sub-regular space, there exists $\lambda \in M(L)$, and there are $P \in \eta^{-}\left(x_{\lambda}\right)$ and $Q \in \eta^{-}\left(\lambda \chi_{E}\right)$ such that $P \vee Q=\top_{X}$.

Let

$$
U=\left\{z: P^{\prime}(z) \not \leq \lambda^{\prime}\right\}, V=\left\{z: Q^{\prime}(z) \not \leq \lambda^{\prime}\right\} .
$$

It is easy to verify that $x \in U, E \subseteq V$ and $U \cap V=\varnothing(U, V \in \mathcal{T})$. Therefore, $(X, \mathcal{T})$ is a regular space.

Case $i=4$ : the proof is similar to that of the case $i=3$.

Now we consider the heredity of the sub-separation. The following results show that sub- $T_{i}\left(i=1,2,2 \frac{1}{2}\right)$ separation axioms are hereditary. Firstly, the concept of the extension is introduced. Let $Y \subseteq X, A \in L^{Y}$. $A^{*} \in L^{X}$ is defined as follows: $\forall x \in X$,

$$
A^{*}(x)= \begin{cases}A(x), & x \in Y . \\ 0, & x \notin Y .\end{cases}
$$

Then $A^{*}$ is called the extension of $A$.

Theorem 3.2. Let $\left(L^{X}, \delta\right)$ be $L$-ts and $Y$ be a nonempty crisp subset of $\mathrm{X}$. If $\left(L^{X}, \delta\right)$ is a sub- $T_{i}$ space, then the subspace $\left(L^{Y}, \delta \mid Y\right)$ is also a sub- $T_{i}$ space, where $\delta \mid Y=\{G \mid Y: G \in \delta\}, i=1,2,2 \frac{1}{2}$.

Proof. We only prove the case $i=2$ and $i=2 \frac{1}{2}$.

Case $i=2$ : Let $x, y \in Y$ with $x \neq y$. Since $\left(L^{X}, \delta\right)$ is a sub- $T_{2}$ space, there exists $\lambda \in M(L)$, and there are $P \in \eta^{-}\left(x_{\lambda}{ }^{*}\right)$ and $Q \in \eta^{-}\left(y_{\lambda}{ }^{*}\right)$ such that $P \vee Q=\top_{X}$, where $x_{\lambda}^{*}, y_{\lambda}^{*}$ are the extensions of $x_{\lambda}, y_{\lambda}$, respectively. Notice that $P \in \eta^{-}\left(x_{\lambda}{ }^{*}\right)$ implies that $P \mid Y \in \eta^{-}\left(x_{\lambda}\right)$. Similarly, $Q \mid Y \in$ $\eta^{-}\left(y_{\lambda}\right)$. Therefore, there exists $\lambda \in M(L)$ and there are $P \mid Y \in \eta^{-}\left(x_{\lambda}\right)$ and $Q \mid Y \in \eta^{-}\left(y_{\lambda}\right)$ such that $(P \mid Y) \vee(Q \mid Y)=\top_{Y}$, i.e., $\left(L^{Y}, \delta \mid Y\right)$ is also a sub- $T_{2}$ space.

Case $i=2 \frac{1}{2}$ : Suppose that $x, y \in Y$ with $x \neq y$. Since $\left(L^{X}, \delta\right)$ is a sub- $T_{2 \frac{1}{2}}$ space, there exists $\lambda \in M(L)$, and there are $P \in \eta^{-}\left(x_{\lambda}^{*}\right)$ and $Q \in \eta^{-}\left(y_{\lambda}^{*}\right)$ such that $P^{\circ} \vee Q^{\circ}=\top_{X}$, where $x_{\lambda}^{*}, y_{\lambda}^{*}$ is the extensions of $x_{\lambda}, y_{\lambda}$, respectively. Noticing that $P \in \eta^{-}\left(x_{\lambda}^{*}\right)$ implies that $P \mid Y \in \eta^{-}\left(x_{\lambda}\right)$ and $Q \in \eta^{-}\left(y_{\lambda}^{*}\right)$ implies that $Q \mid Y \in \eta^{-}\left(y_{\lambda}\right)$, then we have that there exists $\lambda \in M(L)$ and there are $P \mid Y \in \eta^{-}\left(x_{\lambda}\right)$ and $Q \mid Y \in \eta^{-}\left(y_{\lambda}\right)$. Hence we need only to show that $(P \mid Y)^{\circ} \vee(Q \mid Y)^{\circ}=\top_{Y}$. In fact, from $(P \mid Y)^{\circ} \geq\left(P^{\circ} \mid Y\right)$, 
we get that

$$
(P \mid Y)^{\circ} \vee(Q \mid Y)^{\circ} \geq\left(P^{\circ} \mid Y\right) \vee\left(Q^{\circ} \mid Y\right)=\top_{Y} .
$$

Thus $\left(L^{Y}, \delta \mid Y\right)$ is also a sub- $T_{2} \frac{1}{2}$ space.

Theorem 3.3. Let $\left(L^{X}, \delta\right)$ be $L$-ts,$Y$ be a nonempty crisp subset of $\mathrm{X}$ and $\chi_{Y} \in \delta^{\prime}$. If $\left(L^{X}, \delta\right)$ is a sub- $T_{i}$ space, then the subspace $\left(L^{Y}, \delta \mid Y\right)$ is also a sub- $T_{i}$ space, where $i=3,4$.

Proof. We only prove this theorem only for the case $i=3$. Since sub- $T_{1}$ separation axiom is hereditary, we prove the theorem only for the subregular case.

Let $B$ be a nonempty pseudo-crisp closed set in $\left(L^{Y}, \delta \mid Y\right)$ and $y \in Y$ with $y \notin \operatorname{supp} B$. Since $B \in(\delta \mid Y)^{\prime}=\delta^{\prime} \mid Y$, there exists $A \in \delta^{\prime}$ such that $B=A \mid Y$. And we have $B=B^{*} \mid Y$, where $B^{*}$ is the extension of $\mathrm{B}$. It is easy to prove that $B^{*}=A \wedge \chi_{Y}$ and $B^{*}$ is a nonempty pseudo-crisp closed set. By the sub-regularity of $\left(L^{X}, \delta\right)$ and $y \notin \operatorname{supp} B^{*}$, there exists $\lambda \in M(L)$ , and there are $P \in \eta^{-}\left(y_{\lambda}^{*}\right)$ and $Q \in \eta^{-}\left(\lambda B^{*}\right)$ such that $P \vee Q=\top_{X}$, where $y_{\lambda}^{*}$ is the extension of $y_{\lambda}$. Then we know that $P \mid Y \in \eta^{-}\left(y_{\lambda}\right)$ and $Q \mid Y \in \eta^{-}(\lambda B)$ such that $(P \mid Y) \vee(Q \mid Y)=\top_{Y}$. This shows $\left(L^{Y}, \delta \mid Y\right)$ is also a sub-regular space. Therefore, the proof of the theorem is complete.

In the end of this section, we show that sub- $T_{i}\left(i=1,2,2 \frac{1}{2}\right)$ separation axioms are productive. First, a lemma is needed.

Lemma 3.4. Let $\left(L^{X}, \delta\right),\left(L^{Y}, \mu\right)$ be two $L$-ts, $f^{\rightarrow}:\left(L^{X}, \delta\right) \rightarrow\left(L^{Y}, \mu\right)$ be a closed bijection and $f \rightarrow, f^{\leftarrow}$ be continuous. If $\left(L^{X}, \delta\right)$ is a sub-T $T_{i}$ space, then so is $\left(L^{Y}, \mu\right)$, where $i=1,2,2 \frac{1}{2}, 3,4$.

Proof. We prove the theorem only for the case $i=1$ and $i=3$.

Case $i=1$ : For any $y, z \in Y$ with $y \neq z$, since $f$ is a closed bijection, there are $u, v \in X$ with $u \neq v$ such that $f(u)=y, f(v)=z$. Since $\left(L^{X}, \delta\right)$ is a sub- $T_{1}$ space, there exists $\lambda \in M(L)$, both there is $P \in \eta^{-}\left(u_{\lambda}\right)$ such that $v_{\lambda} \leq P$ and there is $Q \in \eta^{-}\left(v_{\lambda}\right)$ such that $u_{\lambda} \leq Q$. Therefore,

$$
\begin{gathered}
y_{\lambda}=f^{\rightarrow}\left(u_{\lambda}\right) \not \leq f^{\rightarrow}(P), z_{\lambda}=f^{\rightarrow}\left(v_{\lambda}\right) \leq f^{\rightarrow}(P), \text { and } \\
z_{\lambda}=f^{\rightarrow}\left(v_{\lambda}\right) \not \leq f \rightarrow(Q), y_{\lambda}=f^{\rightarrow}\left(u_{\lambda}\right) \leq f^{\rightarrow}(Q) .
\end{gathered}
$$

From $P, Q \in \delta^{\prime}$ and $f^{\leftarrow}$ is continuous, we have $f^{\rightarrow}(P) \in \eta^{-}\left(y_{\lambda}\right)$ and $f^{\rightarrow}(Q) \in \eta^{-}\left(z_{\lambda}\right)$. Hence, $\left(L^{Y}, \mu\right)$ is a sub- $T_{1}$ space. 
Case $i=3$ : For any $y \in Y$ and nonempty pseudo-crisp closed set $A \in \mu^{\prime}$ with $y \notin \operatorname{supp} A$. Since $f$ is closed bijection, there exist $x \in X, B \in \delta^{\prime}$ such that $f(x)=y, f(B)=A$ i.e., $x=f^{-1}(y), B=f^{\leftarrow}(A)$. We have $B \in \delta^{\prime}$ from $f^{\rightarrow}$ is continuous. It is easy to prove that $B$ is a nonzero pseudo-crisp closed set. From $y \notin \operatorname{supp} A$, we have:

$$
\begin{aligned}
y \notin \operatorname{supp} A \Rightarrow A(y)=\perp & \Rightarrow A(f(x))=\perp(f(x)=y) \\
& \Rightarrow f^{\leftarrow}(A)(x)=\perp\left(\text { by the definition of } f^{\leftarrow}\right) \\
& \Rightarrow x \notin \operatorname{supp} f^{\leftarrow}(A)=\operatorname{supp} B .
\end{aligned}
$$

For $x \in X$ and $B \in \delta^{\prime}$ with $x \notin \operatorname{supp} B$, since $\left(L^{X}, \delta\right)$ is a sub- $T_{3}$ space, there exists $\lambda \in M(L)$, and there are $P \in \eta^{-}\left(x_{\lambda}\right)$ and $Q \in \eta^{-}(\lambda B)$ such that $P \vee Q=\top_{X}$.

Therefore, $y_{\lambda}=f^{\rightarrow}\left(x_{\lambda}\right) \not \leq f^{\rightarrow}(P), \lambda A=\lambda f^{\rightarrow}(B) \not \leq f^{\rightarrow}(Q), f^{\rightarrow}(P) \vee$ $f^{\rightarrow}(Q)=\top_{Y}$. From $P \in \delta^{\prime}$ and $f^{\leftarrow}$ is continuous, we have $f^{\rightarrow}(P) \in \eta^{-}\left(y_{\lambda}\right)$ and $f^{\rightarrow}(Q) \in \eta^{-}(\lambda A)$. Thus, $\left(L^{Y}, \mu\right)$ is a sub- $T_{3}$ space.

Theorem 3.5. Let $\left\{\left(L^{X_{t}}, \delta_{t}\right)\right\}_{t \in T}$ be a family of $L$-ts and $\left(L^{X}, \delta\right)$ be a product topological space. If for any $t \in T,\left(L^{X_{t}}, \delta_{t}\right)$ is a sub- $T_{i}$ space, then so is $\left(L^{X}, \delta\right)$. If $\left(L^{X}, \delta\right)$ is a sub- $T_{i}$ space and $\left(L^{X_{t}}, \delta_{t}\right)$ is a fully stratified space, then so is $\left(L^{X_{t}}, \delta_{t}\right)$, where $i=1,2,2 \frac{1}{2}$.

Proof. We only prove the case $i=2$, other cases are obtained in the similar way.

Necessity. Suppose that $\left\{\left(L^{X_{t}}, \delta_{t}\right)\right\}_{t \in T}$ is a family of sub- $T_{2}$ space. Let $\forall x=\left\{x^{t}\right\}_{t \in T}, \quad y=\left\{y^{t}\right\}_{t \in T} \in X$ with $x \neq y$, then there exists a $r \in T$ such that $x^{r} \neq y^{r}$. Since $\left(L^{X_{r}}, \delta_{r}\right)$ is a sub- $T_{2}$ space, there exists $\lambda \in$ $M(L)$, and there are $B_{r} \in \eta^{-}\left(x_{\lambda}^{r}\right), \quad C_{r} \in \eta^{-}\left(y_{\lambda}^{r}\right)$ such that $B_{r} \vee C_{r}=$ $\top_{X_{r}}$. Clearly, $P_{r} \leftarrow\left(B_{r}\right), P_{r} \leftarrow\left(C_{r}\right) \in \delta^{\prime}, P_{r} \leftarrow\left(B_{r}\right)(x)=B_{r}\left(x^{r}\right) \nsucceq \lambda$ and $P_{r} \leftarrow\left(C_{r}\right)(y)=C_{r}\left(y^{r}\right) \nsupseteq \lambda$. Furthermore, $x_{\lambda} \not \subset P_{r}^{\leftarrow}\left(B_{r}\right), y_{\lambda} \not \leq P_{r}^{\leftarrow}\left(C_{r}\right)$ and $P_{r} \leftarrow\left(B_{r}\right) \vee P_{r} \leftarrow\left(C_{r}\right)=\top_{X}$. Hence, we prove that $\left(L^{X}, \delta\right)$ is a sub- $T_{2}$ space.

Sufficiency. Let $\left(L^{X}, \delta\right)$ be a sub- $T_{2}$ space and $\left(L^{X_{r}}, \delta_{r}\right)$ be a fully stratified space, where $r \in T$. For any $x=\left\{x^{t}\right\}_{t \in T} \in X$, from Theorem 2.8.9 (Wang [16]), $\left(L^{\tilde{X}_{r}}, \delta \mid \tilde{X}_{r}\right)$ which is parallel to $\left(L^{X_{r}}, \delta_{r}\right)$ through $x$ is homeomorphic to $\left(L^{X_{r}}, \delta_{r}\right)$. Since $\left(L^{\tilde{X}_{r}}, \delta \mid \tilde{X}_{r}\right)$ is a sub- $T_{2}$ space as a subspace of $\left(L^{X}, \delta\right),\left(L^{X_{r}}, \delta_{r}\right)$ is a sub- $T_{2}$ space from Lemma 3.4. 
The next result follows from the above Theorem.

Corollary 3.6. Let $\left\{\left(L^{X_{t}}, \omega_{L}\left(\mathcal{T}_{t}\right)\right)\right\}_{t \in T}$ be a family of $L$-ts topologically generated by a family of topological spaces $\left\{\left(X_{t}, \mathcal{T}_{t}\right)\right\}_{t \in T}$ and $\left(L^{X}, \omega_{L}(\mathcal{T})\right)$ be a product $L$-ts of $\left\{\left(L^{X_{t}}, \omega_{L}\left(\mathcal{T}_{t}\right)\right)\right\}_{t \in T}$. Then $\left(L^{X}, \omega_{L}(\mathcal{T})\right)$ is a sub-T space iff for $\forall t \in T,\left(L^{X_{t}}, \omega_{L}\left(\mathcal{T}_{t}\right)\right)$ is a sub-T space, where $\mathcal{T}=\prod_{t \in T} \mathcal{T}_{t}, i=$ $1,2,2 \frac{1}{2}$.

\section{The relations with respect to other separation axioms.}

In this section, we make a comparison between separation axioms defined in this paper and those presented by Chen and Meng [2], Fang and Ren [3], Gu and Zhao [4], Ganguly and Saha [5], Kubiak [7], Kandil and El-Shafee [8], Shi [14],Shi and Chen [15] and Wang [16], and offer a lot of examples to show the relations between them. At first, we show that the sub-separation axioms defined in this paper are harmonious.

From Definition 2.1, the following theorem is obvious .

Theorem 4.1. Let $\left(L^{X}, \delta\right)$ be an $L$-ts. Then the following implications hold:

(1) $\operatorname{sub}-T_{1} \Rightarrow$ sub- $T_{0}$

(2) sub- $T_{4} \Rightarrow$ sub- $T_{3} \Rightarrow$ sub- $T_{2}$

Theorem 4.2. Let $\left(L^{X}, \delta\right)$ be an $L$-ts. Then sub- $T_{2} \Rightarrow$ sub- $T_{1}$.

Proof. Suppose that $\left(L^{X}, \delta\right)$ is a sub- $T_{2}$ space. For any $x, y \in X$ with $x \neq y$, since $\left(L^{X}, \delta\right)$ is a sub- $T_{2}$ space, there exists $\lambda \in M(L)$, and there are $P \in \eta^{-}\left(x_{\lambda}\right)$ and $Q \in \eta^{-}\left(y_{\lambda}\right)$ such that $P \vee Q=\top_{X}$. From $y_{\lambda} \leq P \vee Q$ and $y_{\lambda} \not \leq Q$, we have $y_{\lambda} \leq P$. Similarly, on account of $x_{\lambda} \leq P \vee Q$ and $x_{\lambda} \not \leq P$, then $x_{\lambda} \leq Q$. Hence, for any $x \in X$ with $x \neq y$, there exists $\lambda \in M(L)$, both there is $P \in \eta^{-}\left(x_{\lambda}\right)$ such that $y_{\lambda} \leq P$ and there is $Q \in \eta^{-}\left(y_{\lambda}\right)$ such that $x_{\lambda} \leq Q$, i.e., $\left(L^{X}, \delta\right)$ is a sub- $T_{1}$ space.

From Theorem 4.1 and 4.2, we obtain the following result that shows the sub-separation axioms are harmonious.

Corollary 4.3. sub- $T_{4} \Rightarrow$ sub- $T_{3} \Rightarrow$ sub- $T_{2} \Rightarrow$ sub- $T_{1} \Rightarrow$ sub- $T_{0}$. 
Theorem 4.4. Let $\left(L^{X}, \delta\right)$ be an $L$-ts. Then sub- $T_{2} \frac{1}{2} \Rightarrow$ sub- $T_{2}$.

Proof. Let $\left(L^{X}, \delta\right)$ be a sub- $T_{2} \frac{1}{2}$ space. For any $x, y \in X$ with $x \neq y$, since $\left(L^{X}, \delta\right)$ is a sub- $T_{2} \frac{1}{2}$ space, there exists $\lambda \in M(L)$, and there are $P \in \eta^{-}\left(x_{\lambda}\right)$ and $Q \in \eta^{-}\left(y_{\lambda}\right)$ such that $P^{\circ} \vee Q^{\circ}=\top_{X}$. Noticing that $P^{\circ} \vee Q^{\circ} \leq P \vee Q$, we get that $P \vee Q=\top_{X}$. Therefore, $\left(L^{X}, \delta\right)$ is a sub- $T_{2}$ space.

The following example shows that the $L$-unit interval $I(L)$ need not satisfy the sub- $T_{1}$ axiom.

Example 4.5. The $[0,1]$-real line $[0,1](I)$ does not satisfy sub- $T_{1}$ axiom. In fact, take $x, y \in[0,1](I)$ satisfying $\forall t \in R$

$$
x(t)=\left\{\begin{array}{ll}
1, & t \in(-\infty, 0), \\
0.5, & t \in[0,1], \\
0, & t \in(1,+\infty),
\end{array} \quad y(t)= \begin{cases}1, & t \in(-\infty, 0), \\
0.6, & t \in[0,1], \\
0, & t \in(1,+\infty)\end{cases}\right.
$$

For convenience, we only consider $P$ or $Q$ which has the forms of $R_{s}^{\prime} \vee L_{t}^{\prime}$. Case I: When $0<\lambda \leq 0.4$, we have that

$$
\begin{gathered}
\varepsilon\left(x_{\lambda}\right)=\vee\left\{t \mid x_{\lambda} \leq L_{t}^{\prime}\right\}=\vee\{t \mid \lambda \leq x(t-)\}=1 \\
\sigma\left(x_{\lambda}\right)=\wedge\left\{s \mid x_{\lambda} \leq R_{s}^{\prime}\right\}=\wedge\left\{s \mid \lambda \leq x(s+)^{\prime}\right\}=0 .
\end{gathered}
$$

Hence,

$$
x_{\lambda} \not \leq L_{t}^{\prime} \vee R_{s}^{\prime} \Rightarrow t>1, s<0 .
$$

Then we get that

$$
\left(L_{t}^{\prime} \vee R_{s}^{\prime}\right)(y)=0 \text { from } t>1, s<0 .
$$

Naturally, we obtain that $y_{\lambda} \not \leq L_{t}^{\prime} \vee R_{s}^{\prime}$.

Case II: When $0.4<\lambda \leq 0.5$, we have that

$$
\varepsilon\left(x_{\lambda}\right)=\vee\left\{t \mid x_{\lambda} \leq L_{t}^{\prime}\right\}=1 ; \quad \sigma\left(x_{\lambda}\right)=\wedge\left\{s \mid x_{\lambda} \leq R_{s}^{\prime}\right\}=0 .
$$

Hence,

$$
x_{\lambda} \not \leq L_{t}^{\prime} \vee R_{s}^{\prime} \Rightarrow t>1, s<0 \text {. }
$$


Then we get that

$$
\left(L_{t}^{\prime} \vee R_{s}^{\prime}\right)(y)=0 \text { from } t>1, s<0 \Rightarrow y_{\lambda} \not \leq L_{t}^{\prime} \vee R_{s}^{\prime} .
$$

Case III: When $0.5<\lambda \leq 0.6$, we have that

$$
\varepsilon\left(y_{\lambda}\right)=\vee\left\{t \mid x_{\lambda} \leq L_{t}^{\prime}\right\}=1 ; \quad \sigma\left(y_{\lambda}\right)=\wedge\left\{s \mid y_{\lambda} \leq R_{s}^{\prime}\right\}=1 .
$$

Hence,

$$
y_{\lambda} \not \leq L_{t}^{\prime} \vee R_{s}^{\prime} \Rightarrow t>1, s<1 .
$$

Then we get that

$$
\left(L_{t}^{\prime} \vee R_{s}^{\prime}\right)(x) \leq 0 \vee 0.5=0.5 \text { from } t>1, s<1 \Rightarrow x_{\lambda} \not \leq L_{t}^{\prime} \vee R_{s}^{\prime} .
$$

Case IV: When $0.6<\lambda \leq 1$, we have that

$$
\varepsilon\left(x_{\lambda}\right)=\vee\left\{t \mid x_{\lambda} \leq L_{t}^{\prime}\right\}=0 ; \quad \sigma\left(x_{\lambda}\right)=\wedge\left\{s \mid x_{\lambda} \leq R_{s}^{\prime}\right\}=1 .
$$

Hence,

$$
x_{\lambda} \not \leq L_{t}^{\prime} \vee R_{s}^{\prime} \Rightarrow t>0, s<1 .
$$

Then we get that

$$
\left(L_{t}^{\prime} \vee R_{s}^{\prime}\right)(y) \leq 0.5 \vee 0.4=0.5 \text { from } t>0, s<1 \Rightarrow y_{\lambda} \not \leq L_{t}^{\prime} \vee R_{s}^{\prime} .
$$

From case I, II, III and IV, we have that $[0,1](I)$ does not satisfy the sub- $T_{1}$ axiom.

Remark 4.6. From the above example, we know that the $L$-unit interval need not satisfy the sub- $T_{1}$ axiom. So the $L$-unit interval is not compatible with the sub-separation axioms proposed in this paper.

Next we make a comparison between the sub-separation axioms and those presented by Kubiak [7].

Theorem 4.7. Let $\left(L^{X}, \delta\right)$ be an $L$-ts. Then sub- $T_{1} \Rightarrow$ Kubiak- $T_{1}$.

Proof. Let $\left(L^{X}, \delta\right)$ be sub- $T_{1}$. In order to prove that $\left(L^{X}, \delta\right)$ is Kubiak$T_{1}$, take $x, y \in X$ with $x \neq y$. Then there exists $\lambda \in M(L)$, and there are $P \in \eta^{-}\left(x_{\lambda}\right)$ and $Q \in \eta^{-}\left(y_{\lambda}\right)$ such that $y_{\lambda} \leq P, x_{\lambda} \leq Q$, respectively. Taking $U=P^{\prime}, V=Q^{\prime}$, we have that $U, V \in \delta, U(x) \not \leq U(y)$ and 
$V(y) \not \leq V(x)$. Therefore, $\left(L^{X}, \delta\right)$ is Kubiak- $T_{1}$.

In general, Kubiak- $T_{1}$ need not imply our sub- $T_{1}$. This can be seen from the following example.

Example 4.8. Let $L=\{\perp, a, b, \top\}$ satisfy $a \vee b=\top, a \wedge b=\perp, a^{\prime}=b$ and $X=\{x, y\}$ with $x \neq y$. Take $\delta=\left\{\perp_{X}, \top_{X}, x_{a}, y_{b}, x_{a} \vee y_{b}\right\}$, then $\delta^{\prime}=\left\{\perp_{X}, \top_{X}, M, N, R\right\}$, where $M, N$ and $R$ are defined as follows:

$$
M(x)=b, M(y)=\top ; \quad N(x)=\top, N(y)=a ; \quad R(x)=b, R(y)=a .
$$

We can prove that $\left(L^{X}, \delta\right)$ is not sub- $T_{1}$, but it is Kubiak- $T_{1}$. Now we show that $\left(L^{X}, \delta\right)$ is not sub- $T_{1}$. We need to show that $\forall \lambda \in M(L)$, $\forall P \in \eta^{-}\left(x_{\lambda}\right)$ such that $y_{\lambda} \not \leq P$, or $\forall Q \in \eta^{-}\left(y_{\lambda}\right)$ such that $x_{\lambda} \not \leq Q$. In fact, we have that $P \in \eta^{-}\left(x_{\lambda}\right)=\left\{\perp_{X}, M, R\right\}, Q \in \eta^{-}\left(y_{\lambda}\right)=\left\{\perp_{X}\right\}$ when $\lambda=a$. Hence we get that $x_{\lambda} \not \subset Q$. Similarly, we have that $P \in \eta^{-}\left(x_{\lambda}\right)=\left\{\perp_{X}\right\}$, $Q \in \eta^{-}\left(y_{\lambda}\right)=\left\{\perp_{X}, N, R\right\}$ when $\lambda=b$. Then we obtain that $y_{\lambda} \not \leq P$. Therefore $\left(L^{X}, \delta\right)$ is not sub- $T_{1}$. Next we show that $\left(L^{X}, \delta\right)$ is Kubiak$T_{1}$. Taking $U=x_{a}, V=y_{b}$, we get that $U, V \in \delta, U(x) \not \leq U(y)$ and $V(y) \not \leq V(x)$.

Theorem 4.9. Let $\left(L^{X}, \delta\right)$ be an $L$-ts and $\top$ be a molecule. Then sub- $T_{2}$ $\Rightarrow$ Kubiak- $T_{2}$.

Proof. For any $x, y \in X$ with $x \neq y$, since $\left(L^{X}, \delta\right)$ is a sub- $T_{2}$ space, there exists $\lambda \in M(L)$, there are $P \in \eta^{-}\left(x_{\lambda}\right)$ and $Q \in \eta^{-}\left(y_{\lambda}\right)$ such that $P \vee Q=\top_{X}$. Taking $U=P^{\prime}, V=Q^{\prime}$, we have that $U, V \in \delta, U(x) \not \subset U(y)$ and $V(y) \not Z V(x)$. Now we only need to prove that $U \leq V^{\prime}$ i.e. $P^{\prime} \leq Q$. In fact, since $P \vee Q=\top_{X}$ and $\top$ is a molecule, we have that $P(x)=\top$ or $Q(x)=\top$ for $\forall x \in X$. Easily we get $P^{\prime} \leq Q$ i.e. $U \leq V^{\prime}$. Therefore $\left(L^{X}, \delta\right)$ is a Kubiak- $T_{2}$ space.

Remark 4.10. If $T$ is not a molecule, is Theorem 4.9 true? We leave it as an open problem. Generally, Kubiak- $T_{2}$ need not imply sub- $T_{2}$ (seeing Example 4.11 below).

Example 4.11. Let $L=[0,1]$ and $X=\{x, y\}$. Take $\delta=\left\{\perp_{X}, \top_{X}, A, B, 0.3^{*}\right.$, $\left.0.7^{*}\right\}$, where $A, B$ are defined as follows: 


$$
A(x)=0.7, A(y)=0.3 ; \quad B(x)=0.3, B(y)=0.7
$$

Then we get that $\left(L^{X}, \delta\right)$ is a Kubiak- $T_{2}$ space. In fact, taking $U=$ $A, V=B$, we get that $U(x) \not \leq U(y) V(y) \not \leq V(x)$ and $U \leq V^{\prime}$. It is easy to check that $\left(L^{X}, \delta\right)$ is not a sub- $T_{2}$ space. For $\forall \lambda \in M(L)$, we get that $\eta^{-}\left(x_{\lambda}\right), \eta^{-}\left(y_{\lambda}\right) \subseteq\left\{\perp_{X}, A, B, 0.3^{*}, 0.7^{*}\right\}$. Therefore for $\forall P \in \eta^{-}\left(x_{\lambda}\right)$, $\forall Q \in \eta^{-}\left(y_{\lambda}\right)$, we have $P \vee Q \leq 0.7^{*}$. Hence $\left(L^{X}, \delta\right)$ is not a sub- $T_{2}$ space. $\square$

Now, we discuss the relation between the sub-separation axioms and other separation axioms presented by Shi[14], Wang[16], Gu and Zhao[4]. The following two examples show that sub- $T_{2}$ need not imply $L-T_{2}$ and $L-T_{2}$ also need not imply sub- $T_{2}$.

Example 4.12. Let $L=[0,1]$ and $X=\{x, y\}$. Take $\delta=\left\{\perp_{X}, \top_{X}, C_{1}, C_{2}, C_{1} \vee\right.$ $\left.C_{2}\right\}$, where $C_{i}$ is defined as follows:

$$
C_{1}(x)=0.5, C_{1}(y)=0 ; \quad C_{2}(x)=0, C_{2}(y)=0.5 .
$$

Easily we get that $\left(L^{X}, \delta\right)$ is a sub- $T_{2}$ space. In fact, taking $\lambda=\frac{2}{3}, P=C_{1}^{\prime}$ and $Q=C_{2}^{\prime}$, we get that $P \in \eta^{-}\left(x_{\lambda}\right), Q \in \eta^{-}\left(y_{\lambda}\right)$ and $P \vee Q=\top_{X}$. It is easy to check that $\left(L^{X}, \delta\right)$ is not $L-T_{2}$.

Example 4.13. Let $L, X$ and $\delta$ be defined as in Example 4.11. From Example 4.11, we know that $\left(L^{X}, \delta\right)$ is not a sub-T $T_{2}$ space. Next we prove that $\left(L^{X}, \delta\right)$ is $L-T_{2}$. Take $Q=A, P=B^{\prime}$, then $Q \in \delta, P \in \delta^{\prime}, Q \leq P$ and $Q(x) \not \leq P(y)$.

Lemma 4.14 (Wang [16]). If $\left(L^{X}, \delta\right)$ is N-compact and $T_{2}$, then it is $T_{4}$.

Obviously, we have the following result.

Theorem 4.15. If $\left(L^{X}, \delta\right)$ is N-compact and $T_{2}$, then it is sub- $T_{i}$, where $i=1,2,2 \frac{1}{2}, 3,4$.

Lemma 4.16 (Gu and Zhao [4]). Let $\left(L^{X}, \delta\right)$ be an $L$-ts. Then,

(1) $\left(L^{X}, \delta\right)$ is Layer $T_{0}$ iff for any $x_{\lambda}, y_{\lambda} \in M\left(L^{X}\right)$ with $x \neq y$, there exists $P \in \delta^{\prime}$ such that $x_{\lambda} \not \leq P$ and $y_{\lambda} \leq P$ or $x_{\lambda} \leq P$ and $y_{\lambda} \not \leq P$.

(2) $\left(L^{X}, \delta\right)$ is Layer $T_{1}$ iff for any $x_{\lambda}, y_{\lambda} \in M\left(L^{X}\right)$ with $x \neq y$, there exists $P \in \delta^{\prime}$ such that $x_{\lambda} \not \leq P$ and $y_{\lambda} \leq P$. 
(3) $\left(L^{X}, \delta\right)$ is Layer $T_{2}$ iff for any $x_{\lambda}, y_{\lambda} \in M\left(L^{X}\right)$ with $x \neq y$, there exist $P, Q \in \delta^{\prime}$ such that $P \in \eta^{-}\left(x_{\lambda}\right), Q \in \eta^{-}\left(y_{\lambda}\right)$ and $P \vee Q \geq[\lambda]$.

By Lemma 4.16, we have the following conclusion.

Theorem 4.17. Let $\left(L^{X}, \delta\right)$ be an $L$-ts. If $\left(L^{X}, \delta\right)$ is a layer $T_{i}$ space, then it is a sub- $T_{i}$ space, where $i=0,1$.

Lemma 4.18 (Gu and Zhao [4]). Let $\left(L^{X}, \delta\right)$ be an $L$-ts. If $\left(L^{X}, \delta\right)$ is a $T_{i}$ space, then it is a layer $T_{i}$ space, where $i=0,1,2$.

Therefore, by Theorem 4.17, Lemmas 4.18, 4.16 (3), the following results hold.

Corollary 4.19. (1) $T_{i} \Rightarrow$ layer $T_{i} \Rightarrow$ sub- $T_{i}$, where $i=0,1$.

(2) layer $T_{2} \Rightarrow$ sub- $T_{2}$ whenever the largest element $T$ is a molecule.

In the following, we give an example showing that a sub- $T_{2}$ space need not be a layer $T_{2}$ space, to say nothing of being $T_{2}$.

Example 4.20. Let $L, X$ and $\delta$ be defined as in Example 4.12. From Example 4.12 , we know that $\left(L^{X}, \delta\right)$ is a sub- $T_{2}$ space. But $\left(L^{X}, \delta\right)$ is not a layer $T_{2}$ space. In fact, taking $\alpha=\frac{1}{3}$, then we get that $\left(X,\left(\tau_{\alpha}\left(\delta^{\prime}\right)\right)^{\prime}\right)=\{\varnothing, X\}$. Clearly, $\left(X,\left(\tau_{\alpha}\left(\delta^{\prime}\right)\right)^{\prime}\right)$ is not a $T_{2}$ space. From Definition 1.5, we know that $\left(L^{X}, \delta\right)$ is not a layer $T_{2}$ space. It is easy to prove that $\left(L^{X}, \delta\right)$ is not a $T_{2}$ space.

From all of examples above, we find that, in general, all sub-separation axioms in this paper are weaker than other separation axioms that had appeared in literature. Indeed, there are many $L$-topological spaces which satisfy sub-separation axioms, but doesn't fulfill other separation axioms. This is one of differences between sub-separation axioms and other separation axioms. For examples, there are good work on separation axioms of $L$-topological spaces in [2], [3], [5] and [8]. In the following, we will offer more examples to show that our sub-separation axioms is very different from separation axioms established in these papers. For simplicity, we only consider $T_{2 \frac{1}{2}}$ and $T_{2}$ separation axiom therein. Recall the definition of $W T_{2}$ in [3] as follows. 
Definition 4.21. An $L$-ts $\left(L^{X}, \delta\right)$ is called a $W T_{2}$-space if for any $x_{\lambda}, y_{\mu} \in$ $M\left(L^{X}\right)$ with $x \neq y$, there are $P \in \eta^{-}\left(x_{\lambda}\right)$ and $Q \in \eta^{-}\left(y_{\mu}\right)$ such that $P \vee Q \geq(\lambda \vee \mu)^{*}$.

Remarks 4.22. (1) For an $L$-ts $\left(L^{X}, \delta\right)$, it is easy to check its $T_{2 \frac{1}{2}}[2]$ (seeing Definition 1.2 introduced in the paper) means sub- $T_{2 \frac{1}{2}}$, but Example 4.23 below shows that the converse needn't be true.

(2) For an $L$-ts $\left(L^{X}, \delta\right)$, it is easy to check its $W T_{2}$ implies sub- $T_{2}$ whenever the largest element $T$ is a molecule, and Example 4.23 below proves that the converse needn't be true.

Example 4.23. Let $L$ be the completely distributive De Morgan algebra having four elements: $\perp, a, b, \top$ satisfying $a \vee b=\top, a \wedge b=\perp, a^{\prime}=b$, and $X=\{x, y\}(x \neq y)$. Take $\delta^{\prime}=\left\{\perp_{X}, \top_{X}, x_{a} \vee y_{b}, x_{b} \vee y_{a}\right\}$. We will show the following conclusions.

(i) The $L$-ts $\left(L^{X}, \delta\right)$ is sub- $T_{2}$. In fact, now $M(L)=\{a, b\}$. For $x, y \in X$ with $x \neq y$, there is $\lambda=a$, and also $P=x_{b} \vee y_{a} \in \eta^{-}\left(x_{\lambda}\right), Q=x_{a} \vee y_{b} \in$ $\eta^{-}\left(y_{\lambda}\right)$ such that $P \vee Q=\top_{X}$. Thus $\left(L^{X}, \delta\right)$ is sub- $T_{2}$, as desired.

(ii) The $L$-ts $\left(L^{X}, \delta\right)$ is sub- $T_{0}$ and also sub- $T_{1}$. These can be obtained by $(i)$ and Theorems 4.1(1), 4.2.

(iii) The $L$-ts $\left(L^{X}, \delta\right)$ isn't $W T_{2}$ in the sense of [3]. Letting $x_{a}, y_{b} \in$ $M\left(L^{X}\right)$, it is easy to check $\eta^{-}\left(x_{a}\right)=\left\{\perp_{X}, x_{b} \vee y_{a}\right\}$ and $\eta^{-}\left(y_{b}\right)=\left\{\perp_{X}, x_{b} \vee y_{a}\right\}$. Hence for any $P \in \eta^{-}\left(x_{a}\right)$ and $Q \in \eta^{-}\left(y_{b}\right)$, it cannot be true that

$$
P \vee Q \geq(a \vee b)^{*}=\top_{X}
$$

where $(a \vee b)^{*}$ is the constant $L$-set with its value $(a \vee b)$ in the sense of [3]. In fact, for any $P \in \eta^{-}\left(x_{a}\right)$ and $Q \in \eta^{-}\left(y_{b}\right), P \vee Q \leq x_{b} \vee y_{a}$, but $x_{b} \vee y_{a} \leq \top_{X}$ and $x_{b} \vee y_{a} \neq \top_{X}$. By Definition 4.21 of $W T_{2}$, the $L$-ts $\left(L^{X}, \delta\right)$ isn't $W T_{2}$.

(iv) The $L$-ts $\left(L^{X}, \delta\right)$ is sub- $T_{2 \frac{1}{2}}$. Let $P=x_{b} \vee y_{a}$ and $Q=x_{a} \vee y_{b}$. It is observed that both $P$ and $Q$ are open since $P^{\prime}=Q$ and $Q^{\prime}=P$. For the unique pair of $x$ and $y$ with $x \neq y$, putting $\lambda=a \in M(L)$, there are $P \in \eta^{-}\left(x_{\lambda}\right)$ and $Q \in \eta^{-}\left(y_{\lambda}\right)$ such that

$$
P^{\circ} \vee Q^{\circ}=P \vee Q=\top_{X}
$$

Thus, $\left(L^{X}, \delta\right)$ is sub- $T_{2 \frac{1}{2}}$ by Definition $2.1(3)$.

$(v)$ The $\left(L^{X}, \delta\right)$ isn't $T_{2 \frac{1}{2}}$ in the sense of [2] (see Definition 1.2 also). 
Indeed, $\eta^{-}\left(x_{a}\right)=\left\{x_{b} \vee y_{a}\right\}$ and $\eta^{-}\left(y_{b}\right)=\left\{x_{b} \vee y_{a}\right\}$. We find that there are two points $x_{a}, y_{b} \in M\left(L^{X}\right)$ with $x \neq y$ such that for any $P \in$ $\eta^{-}\left(x_{a}\right)=\left\{x_{b} \vee y_{a}\right\}$ and $Q \in \eta^{-}\left(y_{b}\right)=\left\{x_{b} \vee y_{a}\right\}$ (it must be $P=x_{b} \vee y_{a}$ and $\left.Q=x_{b} \vee y_{a}\right)$

$$
P^{\circ} \vee Q^{\circ}=P \vee Q=x_{b} \vee y_{a} \neq \top_{X} .
$$

It implies that $\left(L^{X}, \delta\right)$ isn't $T_{2 \frac{1}{2}}$ by Definition 1.2 , as desired.

In [15],Shi and Chen redefined Urysohn in L-topology, where it was called Shi-Urysohn. Now we consider the relation between the Shi-Urysohn axiom and our sub- $T_{2 \frac{1}{2}}$ axiom. Recall the definition of Shi-Urysohn axiom in [15] as follows.

Definition 4.24. (Shi and Chen [15]).

An $L$-ts $\left(L^{X}, \delta\right)$ is said to be Urysohn if any $x_{\lambda}, y_{\mu} \in M\left(L^{X}\right)$ with $x_{\lambda} \not \leq y_{\mu}$, there exist $P \in \eta^{-}\left(x_{\lambda}\right)$ and $Q \in \aleph^{\circ}\left(y_{\mu}\right)$ such that $P^{\circ} \geq Q^{-}$, where $\aleph^{\circ}\left(y_{\mu}\right)=\left\{V: y_{\mu} \leq V, V \in \delta\right\}$.

The following example shows that our sub- $T_{2 \frac{1}{2}}$ need not imply ShiUrysohn.

Example 4.25. Let $X=L=[0,1]$, and $\delta=\left\{\chi_{E}: E \subset X\right\}$, where $\chi_{E}$ is the characteristic function of $\mathrm{E}$. Then $\delta$ is a $[0,1]$-topology on $X$. It is easy to check that $\left(L^{X}, \delta\right)$ is $T_{2 \frac{1}{2}}$ (Urysohn), then it is sub- $T_{2 \frac{1}{2}}$. But it is not Shi-Urysohn. In fact, for any $x \in X$ and any $P \in \eta^{-}\left(x_{\top}\right)$, it follows that $P^{\circ}(x)=\perp$. But there is no $Q \in \aleph^{\circ}\left(x_{0.5}\right)$ such that $P^{\circ} \geq Q^{-}$.

Remark 4.26. Does the Shi-Urysohn axiom imply our sub- $T_{2 \frac{1}{2}}$ ? We can't solve it. So we leave it as an open problem.

To discuss the relation between sub- $T_{2}$ separation axiom and other $T_{2}$ separation axiom introduced in [5] and [8]. Note that we consider the case of $L=I=[0,1]$, the unit interval, so that the conclusions is available for the membership valued lattice using in the published papers [6] and [9]. We introduce some definitions for the convenience of readers.

Definition 4.27. (Liu and Luo[11]). Let $x_{\lambda} \in M\left(I^{X}\right)$ and $A, B \in I^{X}$. We say $x_{\lambda}$ quasi-coincides with $A$, or say $x_{\lambda}$ is quasi-coincident with $A$, denoted 
by $x_{\lambda} q A$, if $A(x)+\lambda>\top$; say $A$ quasi-coincides with $B$ at $x \in X$, or say $A$ is quasi-coincident with $B$ at $x, A q B$ at $x$ for short, if $A(x)+B(x)>\mathrm{T}$; say $A$ quasi-coincides with $B$, or say $A$ is quasi-coincident with $B$, denoted by $A q B$, if $A$ quasi-coincides with $B$ at some point $x \in X$. Relation "does not quasi-coincides with" or "is not quasi-coincident with" is denoted by $\bar{q}$.

Definitio 4.28. (Liu [9]). Let $\left(I^{X}, \delta\right)$ be $I$-ts and $x_{\lambda} \in M\left(I^{X}\right)$. A fuzzy set $U$ is called a quasi-coincident neighborhood (q-nbd, for short) of $x_{\lambda}$ if there exists $V \in \delta$ such that $x_{\lambda} q V$ and $V \leq U$.

Definition 4.29. (C.K. Wong $[16])$. Let $\left(I^{X}, \delta\right)$ be an $I$-ts, $A \in I^{X}$ and $x_{\lambda} \in M\left(I^{X}\right)$.

A is said to be a neighborhood (nbd, in short) in $(X, \delta)$ iff there is a $B \in \delta$ such that $x_{\lambda} \leq B \leq A$. Therefore, an open set $U \in \delta$ is the nbd of each of its points.

Definition 4.30. (S. Ganguly and S. Saha [5]). An $I$-ts $\left(I^{X}, \delta\right)$ is $G S-T_{2}$ (Originally, $T_{2}$ ) iff for any two distinct points $x_{\lambda}$ and $y_{\mu}$ :

Case $I$. When $x \neq y, x_{\lambda}$ and $y_{\mu}$ have open nbds which are not quasicoincident.

Case $I I$. When $x=y$ and $\lambda<\mu$, then $y_{\mu}$ has an open $q$-nbd $V$ and $x_{\lambda}$ has an open nbd $U$ such that $V \bar{q} U$.

Definition 4.31. (A. Kandil and M.E. El-Shafee [8]). An $I$-ts $\left(I^{X}, \delta\right)$ is $F T_{2}$ if $\forall x_{\lambda}, y_{\mu} \in M\left(I^{X}\right)$ with $x_{\lambda} \bar{q} y_{\mu}$, there exist $Q_{x_{\lambda}} \in \delta$ and $Q_{y_{\mu}} \in \delta$ such that

$$
\lambda \leq Q_{x_{\lambda}}(x), \mu \leq Q_{y_{\mu}}(y) \text { and } Q_{x_{\lambda}} \bar{q} Q_{y_{\mu}} .
$$

Remark 4.32. The following Example 4.33 shows that our sub- $T_{2}$ needn't be $G S-T_{2}$ in the sense of Definition 4.30 .

Example 4.33. Let $\left(I^{X}, \delta\right)$ be the $I$-ts defined in Example 4.12. We have showed that $\left(I^{X}, \delta\right)$ is a sub- $T_{2}$ space in Example 4.12. Now we assert that $\left(I^{X}, \delta\right)$ isn't $G S-T_{2}$ in the sense of Definition 4.30. Taking $\lambda=\frac{1}{8}$ and $\mu=\frac{1}{4}$, then $x_{\lambda}$ and $x_{\mu}$ are different points with $\lambda<\mu$. Moreover, $\top_{X}$ is the unique open $Q$-neighborhood of $x_{\mu}$ and the set of open neighborhood of $x_{\lambda}$ is $A=\left\{C_{1}, C_{1} \vee C_{2}, \top_{X}\right\}$. Obviously, for each $V \in A$, we cannot have $V \bar{q} \top_{X}$. Thus, $\left(I^{X}, \delta\right)$ isn't $G S-T_{2}$ in the sense of Definition 4.30 . 
Remark 4.34. The following Example 4.35 shows that our sub- $T_{2}$ needn't be $F T_{2}$ in the sense of Definition 4.31 .

Example 4.35. Let $\left(I^{X}, \delta\right)$ be the $I$-ts defined in Example 4.12. We have showed that $\left(I^{X}, \delta\right)$ is a sub- $T_{2}$ space in Example 4.12. Now we assert that $\left(I^{X}, \delta\right)$ isn't $F T_{2}$. For the $x, y \in X$ with $x \neq y$, taking $\lambda=\frac{1}{3}$, then $x_{\lambda} \bar{q} y_{\lambda}$. The unique neighborhood of $x_{\lambda}$ and $y_{\lambda}$ is $\top_{X}$, moreover $\top_{X} \bar{q} \top_{X}$ never is true. Hence $\left(I^{X}, \delta\right)$ isn't $F T_{2}$.

\section{References}

[1] C. L. Chang, Fuzzy topological spaces, J. Math. Anal. Appl., 24, pp. 182-190, (1968).

[2] S. L. Chen, G. W. Meng, U-separation axioms and characterizations in $L$-fuzzy topological spaces, J. Liaochen. Sci. Technol. Univ., 11(1), pp. 1-6, (1998).

[3] J.X. Fang, B. Ren, A set of new separation axioms in $L$-fuzzy topological spaces, Fuzzy sets and systems, 96, pp. 359-366, (1998).

[4] M. Gu, B. Zhao, Layer separation axioms in $L$-fuzzy topological spaces, Fuzzy Systems and Mathematics, 17, pp. 12-18 (in Chinese), (2003).

[5] S. Ganguly and S. Saha, On separation axioms and $T_{i}$-fuzzy continuity, Fuzzy Sets and Systems, 16, pp. 265-275, (1985).

[6] B. Hutton, Normality in fuzzy topoligical spaces, J. Math. Anal. Appl., 50, pp. 74-79, (1975).

[7] T. Kubiak, On L-Tychonoff spaces, Fuzzy Sets and Systems, 73, pp. 25-53, (1995).

[8] A. Kandil, M.E. El-Shafee, Regularity axioms in fuzzy topological spaces and $F R_{i}$-proximities, Fuzzy Sets and Systems, 27, pp. 217-231, (1988).

[9] Y. Liu, Pointwise characterizations of complete regularity and embeding thorem in fuzzy topological space, Sci. Sinica. Ser. A 26, pp. 138-147, (1983). 
[10] Y. Liu, M. Luo, Separation in latticed induced spaces, Fuzzy Sets and Systems, 36, pp. 55-66, (1990).

[11] Y. Liu, M. Luo, Fuzzy topology, World Scienctific Publishing, Singapore, (1997).

[12] R. Lowen, Fuzzy topological spaces and compactness, J. Math. Anal. Appl., 56, pp. 621-633, (1976).

[13] S. E. Rodabaugh, Categorical frameworks for stone representation theorems, in: S. E. Rodabaugh, et al., (Eds.), Applications of category theory to Fuzzy Subsets, Kluwer Academic Publishers, Netherlands, pp. 177-231, (1992).

[14] F. G. Shi, A new approach to $L-T_{2}, L$-Urysohn, and $L$-completely Hausdorff axioms, Fuzzy Sets and Systems, 157, pp. 794-803, (2006).

[15] F. G. Shi and P. Chen, The Urysohn axiom and the completely Hausdorff axiom in L-topological spaces, Iranian Journal of Fuzzy Systems, Vol. 7, No. 1, pp. 33-45, (2010).

[16] G. Wang, Theory of $L$-fuzzy topolgical spaces, Sha'anxi Normal University Xi'an, (1988) (in Chinese).

[17] C. K. Wong, Fuzzy point and local properties of fuzzy topology, J. Math. Anal. Appl., 46, pp. 316-328, (1974).

[18] P. Wuyts, R. Lowen, On local and global measures of separation in fuzzy topological spaces, Fuzzy Sets and Systems, 19, pp. 51-80, (1986).

[19] F. You, The separation axioms of $T_{2} \frac{1}{2} L$-fts and $S T_{2} \frac{1}{2} L$-fts, Fuzzy Systems and Mathematics, 15, pp. 73-76 (in Chinese), (2001). 
Cui-Mei Jiang

Qingdao Technological University

11 Fushun Road Qingdao 266033

P. R. China

China

e-mail : jiangcuimei2004@163.com

and

Jin-Ming Fang

Department of Mathematics

Ocean University of China

China

e-mail : 